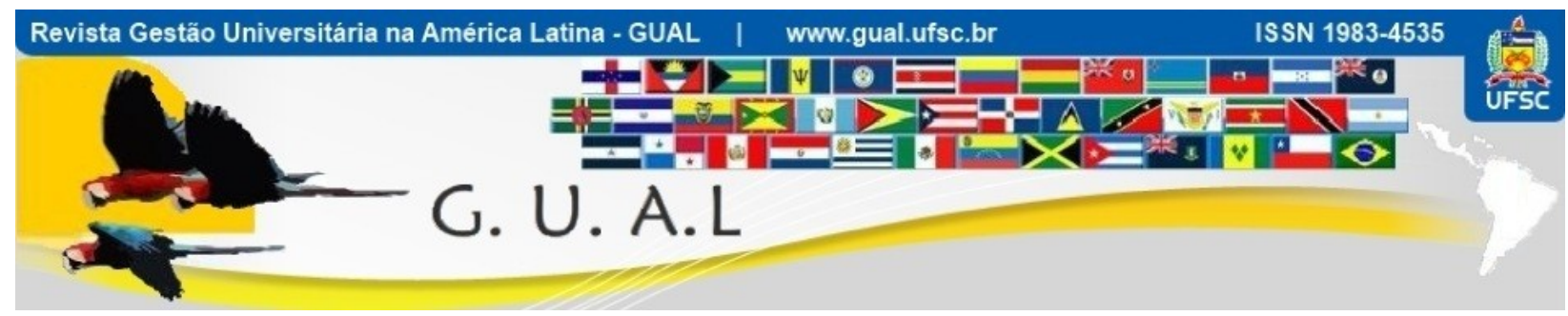

DOI: http://dx.doi.org/10.5007/1983-4535.2015v8n2p216

\title{
AVALIAÇÃO DA QUALIDADE PERCEBIDA EM SERVIÇOS EDUCACIONAIS: PROPOSTA DE UMA ESCALA
}

\section{RATING OF PERCEIVED QUALITY IN EDUCATIONAL SERVICES: PROPOSAL FOR A SCALE}

\author{
Verônica Paludo Bressan, Mestranda \\ Faculdade Meridional - IMED \\ vebressan@gmail.com \\ Kenny Basso, Doutor \\ Faculdade Meridional - IMED \\ kenny@,imed.edu.br \\ Larissa Carine Braz Becker, Mestre \\ Faculdade Meridional - IMED \\ larissamarketing@hotmail.com
}

Recebido em 23/setembro/2014

Aprovado em 08/fevereiro/2015

Sistema de Avaliação: Double Blind Review

Esta obra está sob uma Licença Creative Commons Atribuição-Uso. 


\title{
RESUMO
}

O objetivo geral desta pesquisa é desenvolver e validar uma escala com a finalidade de mensurar a qualidade percebida por alunos de graduação em serviços educacionais. Para sua realização, foi desenvolvida uma pesquisa bibliográfica relacionada aos conceitos de qualidade percebida no ensino e formas de mensuração da qualidade, identificando a necessidade de desenvolvimento de uma escala específica para serviços educacionais, uma vez que as ferramentas encontradas tratavam de serviços de forma genérica. Foram seguidas as etapas propostas por Churchill (1979), sendo realizadas entrevistas com 17 alunos, na etapa qualitativa exploratória, e aplicados questionários em 410 alunos, na etapa quantitativa descritiva, composta por duas amostras, de teste e validação. Os resultados obtidos através de análise fatorial exploratória e análise fatorial confirmatória demonstraram a confiabilidade da escala, permitindo sua aplicação no contexto gerencial das IES. A escala final foi composta por 20 indicadores e 6 dimensões: organização curricular, corpo docente, pesquisa e extensão, organização administrativa, infraestrutura e serviços agregados. Este estudo apresenta implicações acadêmicas, colaborando com a discussão sobre os indicadores que compõem a qualidade percebida em serviços educacionais. Também são identificadas implicações gerenciais, uma vez que apresenta uma nova ferramenta que poderá ser utilizada nas IES como forma de qualificar a entrega dos serviços aos alunos.

Palavras chave: Serviços educacionais. Qualidade em serviços. Mensuração da qualidade. Qualidade no ensino superior.

\begin{abstract}
The aim of this research is to develop and validate a scale in order to measure the quality perceived by undergraduate students in educational services. To carry out the work, was developed a bibliographic research about the concepts and features related to perceived quality, quality in higher education and ways to measure service quality, identifying the need to develop a specific scale for educational services in view of the tools found in the literature treated services in a general terms. To develop the scale so followed the steps suggested by Churchill in 1979, by conducted interviews with 17 students, in the qualitative exploratory research, and applied 410 questionnaires in students in the quantitative descriptive stage, consisting of two samples, for test and for validation. The results obtained by conducting exploratory factor analysis and confirmatory factor analysis demonstrated the reliability of the proposed scale, allowing its application in the context of higher education's managerial. The final scale was composed by 21 indicators, and 6 dimensions: curriculum organization, professors, research and extension learning, administrative organization, infrastructure and additional services. This study has academic's implications, because that collaborates with the discussion and on the indicators that make up the perceived quality of educational services. Also, has a managerial implication, because it presents a new tool that can be used in higher education institutions as a way to qualify the delivery of services to the students.
\end{abstract}

Keywords: Educational services. Quality services. Quality measurement. Quality in higher education. 


\section{INTRODUÇÃO}

Com o aumento da oferta de cursos de graduação, a concorrência entre Instituições de Ensino Superior (IES) apresentou grande crescimento, com uma demanda inferior ao número de vagas ofertadas. Mesmo aquelas IES que conseguem preencher todas as vagas oferecidas nos processos seletivos passam por dificuldades em reter os alunos, atingindo em 2010, de acordo com dados do Instituto Nacional de Estudos e Pesquisas Educacionais (INEP), um índice de 15,6\% de evasão em instituições privadas. Sem conseguir identificar as expectativas do público alvo e sem processos confiáveis para a medição da qualidade percebida pelos alunos, a gestão não é apoiada por ferramentas adequadas.

De acordo com Meyer et al. (2004), pode-se identificar, dentre outras causas dos problemas de gestão nas IES, a falta de ferramentas gerenciais específicas para esse segmento. Conforme Lourenço et al. (2006), dentre as ferramentas de gestão, o instrumento para medição da qualidade desempenha papel importante para aquelas organizações comprometidas com a melhoria de seus serviços e processo.

A fim de medir a qualidade percebida pelos consumidores de serviços, Parasuraman et al. desenvolveram, em 1988, a escala SERVQUAL, composta por 22 itens que têm por objetivo medir cinco dimensões da qualidade: tangibilidade, confiabilidade, segurança, empatia e presteza. Essa escala utiliza a comparação entre as expectativas prévias e a performance percebida pelo cliente na prestação do serviço. Críticas à escala SERVQUAL apontam que, caso as expectativas sejam medidas após a prestação do serviço, o que normalmente ocorre, então essa expectativa foi influenciada pela experiência. Outro fator apontado por Cronin e Taylor (1992) é relacionado ao fato de não existirem evidências teóricas da relevância da lacuna expectativa-qualidade para a medição da qualidade.

Como alternativa à escala SERVQUAL, em 1992, Cronin e Taylor desenvolveram a escala SERVPERF, que se difere por não levar em consideração as expectativas prévias do cliente para a medição da qualidade. Porém, as dimensões e itens avaliados são os mesmos. Importante destacar que na SERVPERF, assim como na SERVQUAL, não há adequação ao segmento em que o serviço avaliado está inserido. Deve ser considerado que os 22 itens, por serem genéricos, não compreendem as especificidades de um determinado tipo de serviço, como é o caso do serviço educacional.

Alguns trabalhos tentaram adaptar estas escalas para o segmento do ensino (SIQUEIRA; CARVALHO, 2006; LOURENÇO et al., 2006; CERCHIARO; MOTA, 2010), 
porém, sem que tenham sido desenvolvidos itens específicos que atendam às particularidades desse segmento. Siqueira e Carvalho (2006) apresentaram uma pesquisa utilizando a escala SERVPERF adaptada ao contexto do ensino superior com enfoque em duas instituições públicas, concluindo que os resultados obtidos não podem ser replicados em instituições privadas, carecendo de novas pesquisas.

No cenário das IES, a qualidade não é percebida pelo aluno somente em sala de aula, mas em todas as atividades relacionadas à educação (SIQUEIRA; CARVALHO, 2006). Diante disso, torna-se relevante desenvolver uma escala que compreenda os diversos indicadores de qualidade percebida utilizados para inferir sobre a qualidade dos respectivos cursos e instituições. Garcia (2006) destaca que, em um cenário com maior competitividade, mais custos e clientes cada vez mais exigentes, a adoção de práticas modernas para a gestão, dentre elas e medição da qualidade percebida, se torna imperativa.

Diante do aumento da concorrência no ensino superior, as instituições objetivam, cada vez mais, a excelência nos serviços prestados aos alunos com a intenção de garantir a retenção, satisfação e boca a boca positivo, além de potencializar seus resultados financeiros. Para atingir este objetivo, é fundamental avaliar a qualidade dos serviços prestados. De acordo com Sachdev e Verma (2004), é possível identificar a relação entre qualidade percebida com a rentabilidade e os custos. Além disso, ainda de acordo com os autores, a qualidade pode ser relacionada à satisfação, retenção dos clientes, intenção comportamental e boca a boca positivo, colaborando com as estratégias de marketing da empresa, contribuindo de forma positiva para seu posicionamento no mercado em que atua.

Diante da relevância acadêmica e prática do tema, o estudo teve por objetivo desenvolver uma escala para a mensuração da qualidade percebida por alunos de graduação em serviços educacionais, para tanto, foram mapeadas as escalas utilizadas para avaliar a qualidade percebida, identificados os fatores que compõem a qualidade percebida por alunos de graduação e verificada as propriedades psicométricas da escala.

\section{QUALIDADE PERCEBIDA}

A preocupação com o tema qualidade não é recente (LAZZARI et al., 2009). De acordo com Cerchiaro e Mota (2010), seu conceito teve início na literatura de marketing associado à produção de bens e produtos (indústria). O termo possui várias definições e, apesar dessas demonstrarem variações de acordo com a análise de cada setor econômico, a 
maioria passa pelo conceito de conformidade, adequação ao uso e atendimento às expectativas e necessidades dos clientes. De forma mais ampla, Zeithaml (1988) informa que a qualidade pode ser definida como a superioridade ou a excelência de determinado serviço ou produto.

Especificamente em relação a serviços, de acordo com Parasuraman et al. (1985), a qualidade é identificada como a discrepância entre as expectativas dos clientes e suas percepções, ou seja, o desempenho percebido pelo consumidor. Lazzari et al. (2009) apontam a importância de identificar o significado de qualidade para o cliente, fazendo com que esse se torne o foco das ações atinentes à qualidade.

Em um estudo realizado em 1985, Parasuraman, Zeithaml e Berry identificaram cinco dimensões que os consumidores utilizam para julgar a qualidade na prestação de serviços. As dimensões da qualidade identificadas pelos autores foram confiabilidade, responsividade, segurança, empatia e aspectos tangíveis.

De acordo com Moura et al. (2007), a entrega de serviços de qualidade é atualmente considerada uma estratégia fundamental para a sobrevivência e sucesso das empresas no cenário competitivo em que estão inseridas. Parasuraman et al. (1988) compartilham dessa opinião ao identificar que a qualidade pode ser considerada uma importante alternativa de diferenciação, sendo essencial que os gestores entendam o impacto gerado pela qualidade dos serviços sobre os lucros e outros resultados econômicos e financeiros de uma empresa.

Além da capacidade de gerar diferenciais competitivos, é possível identificar a relação entre a qualidade percebida em serviços com outras variáveis. A literatura aponta que a qualidade percebida pode gerar no consumidor, comprometimento, lealdade, percepção de valor, satisfação, intenção de recompra, comunicação boca a boca positiva, além de potencializar a lucratividade e reduzir os custos da organização (ZEITHAML, 2000; HENNIG-THURAU et al., 2001; BASSO et al., 2008). Zeithaml (2000) identificou que a qualidade percebida por clientes pode afetar positivamente sua intenção em relação à empresa, fazendo com que demonstrem preferência em detrimento das concorrentes, aumentando o volume de compras e sua disponibilidade de pagar um preço-prêmio.

Especificamente, em relação ao contexto educacional, Hennig-Thurau et al. (2001) identificaram uma forte relação entre a qualidade percebida pelos alunos e sua lealdade em relação à instituição. Acompanhando este resultado, em um estudo realizado com o objetivo de identificar a relação entre a confiança, qualidade percebida e lealdade no contexto 
especifico do ensino superior, Basso et al. (2008) identificaram que a qualidade percebida tem um impacto positivo na lealdade e na confiança dos estudantes.

Além de questões mercadológicas e de gestão que levam as IES a identificar a qualidade percebida por seus alunos, a Constituição da República Federativa do Brasil, no artigo 206, inciso VII, informa que o ensino deverá ser ministrado com a garantia de um padrão de qualidade (BRASIL, 1988). Visando atender a essa exigência legal, o Ministério da Educação (MEC) e o Instituto Nacional de Estudos e Pesquisas Educacionais (INEP) vêm desenvolvendo alternativas para garantir a qualidade de ensino no Brasil através de avaliações técnicas e exames realizados periodicamente com os alunos.

Em face aos diversos e importantes impactos da qualidade percebida pelos consumidores de serviços, pesquisas sobre ferramentas para sua mensuração demonstram-se relevantes. As características específicas dos serviços, em especial dos serviços educacionais, aliado às dificuldades de gestão apontadas, tornam a mensuração da qualidade nesse setor mais complexa, pois está diretamente relacionada às interações entre os alunos e professores.

\subsection{MENSURAÇÃO DA QUALIDADE}

A relevância da mensuração da qualidade em serviços fica evidente na relação realizada por Lopes et al. (2009). De acordo com os autores, a qualidade percebida em serviços está fortemente relacionada à satisfação dos consumidores, sendo essencial para atrair e reter clientes. Estudos vêm sendo realizados a fim de desenvolver ferramentas e técnicas adequadas, que gerem resultados críveis e válidos para a mensuração da qualidade percebida em serviços (MIGUEL; SALOMI, 2004).

Dentre as principais escalas desenvolvidas para mensuração da qualidade percebida em serviços podem-se destacar a SERVQUAL e a SERVPERF. A SERVQUAL foi desenvolvida por Parasuraman et al. (1985; 1988), tendo como base o paradigma da desconfirmação, no qual os clientes comparam o que esperam que o fornecedor deveria oferecer e suas percepções do desempenho real do serviço entregue (PARASURAMAN et al. 1988). Em estudo realizado em 1988 foram identificadas cinco dimensões da qualidade em serviços: tangibilidade, confiabilidade, presteza, segurança e empatia. Essa escala é tida como a mais tradicional ferramenta para a medição da qualidade em serviços (LOPES et al., 2009), tendo sido aplicada diversas vezes, em segmentos diferentes (BANDEIRA et al., 1998; 
MATOS; VEIGA, 2000; LOURENÇO et al., 2006; MOURA et al., 2007; CERCHIARO; MOTA, 2010).

Mesmo com sua popularidade, a SERVQUAL é alvo de críticas operacionais e de mensuração (CERCHIARO; MOTA, 2010), Cronin e Taylor (1994) identificaram que a mensuração das expectativas do consumidor é desnecessária, afirmando que a medição da qualidade percebida é suficiente para apurar o desempenho do prestador de serviços. Outra crítica, realizada por Carman (1990), apontou a dificuldade de medir as expectativas e percepções de um mesmo consumidor, informando não ser prático descrever as expectativas desses e aguardar até o final da prestação do serviço para descrever também suas percepções. Como exemplo, no serviço educacional, as expectativas e percepções podem não ser identificadas em um mesmo consumidor, mas sim, em pessoas diferentes.

A partir destas considerações, a escala SERVPERF foi desenvolvida por Cronin e Taylor (1992), em um modelo semelhante à SERVQUAL, utilizando os mesmos indicadores, porém baseando-se somente na percepção de desempenho dos consumidores, uma vez que os autores afirmam que a qualidade não deve ser medida como a diferença entre expectativas e desempenho, mas como uma percepção, que levará à satisfação dos consumidores. Essa escala vem sendo utilizada em estudos acadêmicos, em diferentes segmentos (SIQUEIRA; CARVALHO, 2006).

No contexto educacional, de acordo com Bandeira et al. (1998), tendo em vista a necessidade das IES se ajustarem às demandas modernas da sociedade, o desenvolvimento de ferramentas e metodologias para avaliar a qualidade no ensino superior emerge como um tema relevante e oportuno.

De acordo com Casagrande (2011), os indicadores oficiais, que atendem às exigências legais impostas pelo MEC e INEP, utilizados para mensurar a qualidade no ensino superior são: (a) o Exame Nacional de Desempenho de Estudantes (ENADE), (b) o Indicador de Diferença entre os Desempenhos Observados e Esperados (IDD), (c) o Conceito Preliminar de Curso (CPC) e (d) o Índice Geral de Cursos (IGC), que se refere a um indicador de qualidade de IES.

Além dos índices apresentados, as IES precisam desenvolver o processo de autoavaliação, através das avaliações institucionais (CASTANHEIRA et al., 2008). Em relação a essa ferramenta, o INEP irá avaliar se a instituição possui uma comissão permanente de avaliação, a abrangência do processo de auto-avaliação, a participação dos integrantes da 
comunidade acadêmica, a divulgação dos resultados obtidos e as ações acadêmicas e administrativas que são implantadas a fim de sanar eventuais problemas identificados (FREITAS; RODRIGUES, 2003). Cabe destacar que a avaliação institucional desenvolvida em grande parte das IES não é um instrumento confiável, por não passar por processos de validação, sendo utilizada erroneamente para medir indicadores como a satisfação e a qualidade percebida pelos alunos.

O estudo sobre a qualidade em ensino vem gerando pesquisas com a finalidade de auxiliar as organizações a identificar os antecedentes da qualidade, investigando os atributos que compõem a qualidade nos serviços educacionais, o que permitirá a criação de ferramentas adequadas para a mensuração da qualidade nesse segmento. Srikanthan e Dalrymple (2003) identificaram que os estudos desenvolvidos sobre a medição da qualidade nas IES têm por hábito focar somente na educação (atividade fim das instituições), não levando em consideração outros fatores que contribuem para a percepção de qualidade por parte dos alunos, presentes na prestação do serviço.

\section{METODOLOGIA DE PESQUISA}

O objetivo geral deste trabalho é a validação e o desenvolvimento de uma escala para mensurar a qualidade percebida por alunos de graduação em IES. Para tanto, foi utilizado o método proposto por Churchill (1979) o qual pretendia apresentar elementos críticos para a evolução do conhecimento relacionado a medidas e à possibilidade de aplicação prática, a partir de oito etapas para o desenvolvimento de escalas, apresentando, também, a recomendação dos coeficientes e técnicas estatísticas apropriados para seu desenvolvimento.

De acordo com a proposta, a primeira etapa está relacionada à construção do instrumento de coleta de dados com base em pesquisas bibliográficas relacionadas à teoria referente ao assunto para o qual a escala está sendo desenvolvida. A segunda etapa refere-se à identificação de uma amostra dos itens que serão avaliados, com base, entre outras ferramentas, em pesquisas literárias, survey ou focus group. A próxima etapa trata da coleta dos dados, para posterior purificação das medidas através de análise fatorial, na sequência, o autor recomenda que seja realizada nova coleta de dados com avaliação da confiabilidade com base no coeficiente alpha ou na confiabilidade split-half, e avaliação da validade com a utilização da matriz de múltiplos métodos ou o critério de validação. $\mathrm{O}$ autor conclui sugerindo o desenvolvimento de normas através de médias e outras medidas estatísticas. 
De acordo com o apresentado duas etapas são necessárias para o desenvolvimento de uma escala, uma qualitativa exploratória e outra quantitativa descritiva. Desta forma, esta pesquisa foi desenvolvida com base nestas duas fases, que serão descritas a seguir.

\subsection{ETAPA QUALITATIVA EXPLORATÓRIA}

Esta etapa teve o objetivo de identificar os itens que compõem a qualidade percebida em ensino por alunos. Foi utilizada uma abordagem qualitativa exploratória de pesquisa (MALHOTRA, 2012). A unidade de análise foi definida por alunos de graduação de duas IES privadas do Rio Grande do Sul. A escolha de duas IES foi feita buscando obter maior variação na avaliação dos entrevistados. Para a escolha dos entrevistados, levou-se em consideração variações de idade, sexo, curso e níveis em que os alunos estão matriculados.

A quantidade de sujeitos entrevistados foi definida pela saturação de termos, em 17 alunos de graduação matriculados em Administração (18\%), Arquitetura e Urbanismo (12\%), Direito (24\%), Engenharia Civil (18\%), Psicologia (12\%), Publicidade em Propaganda (6\%) e Sistemas de Informação (12\%). Dos entrevistados, 27,78\% estão matriculados até o $5^{\circ}$ semestre e $72,22 \%$ encontram-se matriculados no $6^{\circ}$ semestre ou superior. A idade média foi de 22 anos, variando entre 18 e 29 anos, sendo $65 \%$ mulheres e $35 \%$ homens.

Para a coleta dos dados foi utilizada a técnica de entrevista em profundidade. Os alunos foram abordados e convidados pessoalmente para participar das entrevistas individuais. Para as entrevistas, foi utilizado um roteiro de entrevistas, que foi construído tendo como base pesquisas realizadas na literatura disponível sobre o tema da qualidade percebida em serviços (PARASURAMAN et al., 1985; CRONIN; TAYLOR, 1992), buscando também considerar pesquisas com foco em percepção de qualidade em serviços educacionais (BANDEIRA et al., 1998; LOURENÇO et al., 2006). Este roteiro teve sua validação de conteúdo realizada por dois professores pesquisadores da área de marketing. As entrevistas foram transcritas em fichas que foram utilizadas para a análise de conteúdo

A partir da realização das entrevistas, os dados foram tratados a partir da técnica de análise de conteúdo. Para tanto, foi realizada uma leitura exaustiva dos dados coletados e identificados os indicadores de qualidade percebidos pelos alunos, os quais foram transformados em questões que serviram como base para o desenvolvimento do questionário utilizado na próxima etapa, quantitativa descritiva, que será apresentada na sequência. 


\subsection{ETAPA QUANTITATIVA DESCRITIVA}

Essa etapa da pesquisa teve o objetivo de testar e validar os indicadores de qualidade percebida identificados na pesquisa qualitativa exploratória. Para seu desenvolvimento, a abordagem escolhida foi a quantitativo-descritiva. Seguindo o modelo proposto por Churchill (1979), a etapa quantitativa foi desenvolvida em duas amostras distintas e independentes, uma primeira para testar a escala obtida, com a finalidade de reduzir o número de indicadores; e outra amostra, visando validar e verificar a confiabilidade do instrumento final. A população desta etapa é representada por alunos de graduação das mesmas IES utilizadas na etapa anterior. Nesta etapa, optou-se pela técnica de amostragem não-probabilística, por conveniência.

A primeira amostra foi composta por 208 alunos matriculados nos cursos de graduação nos cursos de Administração (23,19\%), Arquitetura e Urbanismo (10,14\%), Direito (22,22\%), Engenharia Civil (1,93\%), Odontologia (11,11\%), Psicologia (19,81\%) e Sistemas de Informação (11,59\%), com média de idade de 23 anos e variação entre 18 e 51 anos, divididos em $62,62 \%$ mulheres e $37,38 \%$ homens. Em relação ao semestre em que estavam matriculados, $53,40 \%$ dos alunos estavam cursando semestres iniciais (até o $5^{\circ}$ semestre) enquanto 46,60\% cursavam semestres finais (a partir do $6^{\circ}$ semestre). Em relação à ocupação profissional, dos respondentes, $75,12 \%$ estavam trabalhando, enquanto $24,88 \%$ informaram estar desempregados.

A segunda amostra, com a finalidade de validação da escala proposta foi composta por 202 estudantes, matriculados nos cursos de Administração (22,89\%), Arquitetura e Urbanismo (8,96\%), Design de Moda (5,97\%), Direito (10,95\%), Engenharia Civil (8,96\%), Psicologia (31,84\%), Publicidade e Propaganda (2,99\%) e Sistemas de Informação (7,46\%). O perfil dos respondentes apresentou grande variação, contando com 63,13\% de mulheres e $36,87 \%$ de homens. Em relação à idade, os alunos com até 25 anos representaram 76,04\% do total de respondentes, a idade média dos respondentes foi de 23 anos e variação entre 17 e 47 anos.

O questionário, composto pelos itens identificados na primeira etapa, foi encaminhado para validação de conteúdo pelos dois professores que haviam realizado a validação do roteiro de entrevistas anteriormente. Após a validação de conteúdo foi realizado o pré-teste com um grupo de 12 pessoas, da mesma população do estudo. O tempo médio de resposta identificado no pré-teste foi de 12 minutos. 
O questionário foi aplicado nos alunos de graduação integrantes da amostra de teste, sendo composto por 65 perguntas relacionadas à qualidade percebida (estas perguntas estão disponíveis em contato com os autores). As perguntas foram mensuradas a partir de uma escala com sete pontos de variação, do tipo Likert (Muito Ruim a Muito Bom). Era perguntado aos sujeitos "Qual é o desempenho de sua Universidade/Faculdade em relação aos seguintes itens". Ao final, o questionário contou com os dados de identificação do respondente: curso, semestre, idade, gênero, trabalho, renda familiar mensal e se possui bolsa de estudos ou crédito educativo.

Após a aplicação e tabulação dos questionários nos alunos integrantes da amostra de teste, a escala foi reduzida através da análise fatorial exploratória, com rotação varimax. $\mathrm{O}$ questionário reduzido, que posteriormente foi aplicado na amostra de validação, foi composto por 20 perguntas na escala de qualidade percebida, além dos indicadores de qualificação da amostra. Após, uma análise fatorial confirmatória foi utilizada para avaliar a confiabilidade e validade da escala. Os dados foram analisados através dos softwares SPSS Statistics e SmartPLS 2.0M3.

\section{RESULTADOS}

\subsection{ANÁLISE FATORIAL EXPLORATÓRIA}

Em um primeiro momento, foram realizadas as análises dos dados obtidos na amostra de teste, com o objetivo de realizar uma redução dos indicadores identificados na etapa qualitativa. Inicialmente, o número de dimensões foi determinado a partir de autovalores (eigenvalue) superiores a 1, que, após a exclusão dos indicadores com comunalidades menores que 0,400 e daqueles que apresentavam cargas cruzadas, gerou um resultado com cinco fatores. Esta solução não contava com nenhuma questão relacionada ao corpo docente. Rossiter (2002) aponta que, a determinação dos indicadores que irão compor uma escala, deve ser realizada levando em consideração o objeto do estudo, incluindo seus componentes. De acordo com Siqueira e Carvalho (2006) em relação aos serviços educacionais, os professores, junto à infraestrutura, integram a dimensão funcional da prestação do serviço, o "como", que está relacionado ao processo e à forma como o serviço é prestado, sendo um dos fatores que integram a percepção de qualidade pelos clientes, considerado mais subjetivo e difícil de mensurar. 
Além da relevância teórica do tema, cabe destacar que os professores foram citados repetidamente durante as entrevistas realizadas na etapa qualitativa deste estudo, sendo citados 40 vezes nas 17 entrevistas realizadas.

A partir destas informações, tendo em vista a relevância dos indicadores relacionados aos professores para a escala proposta, optou-se por realizar a extração a partir de um número fixo, sendo definidos seis fatores. Após a definição deste número, o resultado na análise fatorial obtido com seis dimensões manteve as questões relacionadas a professores: titulação dos professores, professores atualizados com os conhecimentos práticos e ferramentas da profissão e professores preparados para as aulas.

Baseado na extração em seis dimensões, 45 indicadores foram eliminados por apresentarem carga fatorial cruzada em duas ou mais dimensões, e um indicador foi eliminado por apresentar baixa comunalidade (menor que 0,400). A partir da eliminação destes indicadores, foi realizada a análise fatorial exploratória final para a amostra de teste. $\mathrm{Na}$ amostra de teste, o resultado do teste $\operatorname{KMO}(0,861)$ e da esfericidade de Barlett $\left(\chi^{2}=1.458,61\right.$, $g l=190, p<0,001)$ demonstraram sua adequação para a realização da análise fatorial exploratória (HAIR et al., 2009).

Para a solução final da análise fatorial exploratória da amostra de teste, apresentada na Tabela 1, o conjunto de seis dimensões, compostas por 20 indicadores, explica $74,22 \%$ da variação total dos dados da pesquisa.

Em relação às comunalidades, o menor valor obtido foi de 0,612 (estacionamento) e o maior de 0,849 (grupos de estudos). Todos os indicadores apresentaram cargas fatoriais elevadas em cada uma das dimensões $(>0,50)$. Nesta solução fatorial, apenas uma carga cruzada elevada foi diagnosticada, referente à titulação dos professores $(0,416)$, todavia tal carga é menor do que a carga fatorial do indicador na dimensão $6(0,539)$.

Após a apreciação dos resultados obtidos a partir da análise fatorial exploratória realizada na amostra de teste, o questionário foi reduzido, mantendo 20 indicadores de qualidade percebida, que representam os indicadores das seis dimensões. O novo questionário obtido foi aplicado nos 202 alunos, integrantes da amostra de validação. Após a aplicação e tabulação dos dados, os resultados foram tratados com os mesmos procedimentos estatísticos anteriores, obtendo resultado do teste KMO de 0,794 e significância no teste de esfericidade de Barlett $\left(\chi^{2}=1.666,24, g l=190, p<0,001\right)$, demonstrando sua adequação à análise fatorial exploratória (HAIR et al., 2009). 
Tabela 1 Estrutura Fatorial da Amostra de Teste

\begin{tabular}{|c|c|c|c|c|c|c|c|}
\hline \multirow{2}{*}{ Indicadores } & \multicolumn{6}{|c|}{ Dimensões } & \multirow{2}{*}{ Comunalidades } \\
\hline & 1 & 2 & 3 & 4 & 5 & 6 & \\
\hline 1 - Oferta de aulas práticas & 0,152 & 0,713 & 0,168 & 0,187 & 0,092 & 0,131 & 0,620 \\
\hline 2 - Conteúdos $\mathrm{x}$ aulas práticas & 0,129 & $\mathbf{0 , 8 1 9}$ & 0,066 & 0,115 & 0,081 & 0,212 & 0,756 \\
\hline 3 - Adequação do currículo à prática & 0,112 & 0,785 & 0,198 & 0,029 & 0,144 & 0,104 & 0,700 \\
\hline 7 - Ferramentas $\mathrm{p} /$ prática profissional & 0,152 & 0,658 & 0,253 & 0,140 & 0,169 & 0,279 & 0,646 \\
\hline 10 - Serviços à comunidade & 0,121 & 0,169 & 0,787 & 0,074 & 0,164 & 0,039 & 0,697 \\
\hline 11 - Grupos de estudos & 0,201 & 0,162 & $\mathbf{0 , 8 5 8}$ & $-0,030$ & $-0,032$ & 0,212 & 0,849 \\
\hline 12 - Projetos de pesquisa ou extensão & 0,095 & 0,175 & 0,886 & 0,013 & 0,080 & 0,079 & 0,838 \\
\hline 14 - Professores - titulação & 0,164 & 0,299 & 0,416 & 0,176 & $-0,083$ & $\mathbf{0 , 5 3 9}$ & 0,618 \\
\hline 17 - Professores - atualizados & 0,239 & 0,358 & 0,197 & 0,092 & 0,032 & $\mathbf{0 , 7 3 6}$ & 0,774 \\
\hline 19 - Professores - preparados p/ aula & 0,303 & 0,375 & 0,094 & 0,138 & 0,165 & $\mathbf{0 , 6 8 1}$ & 0,751 \\
\hline 22 - Salas de aula & 0,035 & 0,093 & 0,074 & $\mathbf{0 , 8 8 2}$ & 0,027 & 0,106 & 0,805 \\
\hline 23 - Limpeza dos ambientes & 0,125 & 0,124 & 0,024 & $\mathbf{0 , 8 2 0}$ & 0,101 & $-0,055$ & 0,718 \\
\hline 24 - Conforto dos ambientes & 0,231 & 0,166 & $-0,031$ & $\mathbf{0 , 7 4 7}$ & 0,211 & 0,254 & 0,748 \\
\hline 40 - Eficiência do atendimento & $\mathbf{0 , 8 5 4}$ & 0,077 & 0,216 & 0,221 & 0,069 & 0,067 & 0,840 \\
\hline 41 - Comunicação alunos/funcionários & $\mathbf{0 , 8 7 4}$ & 0,134 & 0,132 & 0,106 & 0,131 & 0,089 & 0,836 \\
\hline 44 - Funcionários - educação & 0,768 & 0,135 & $-0,042$ & 0,121 & 0,220 & 0,283 & 0,753 \\
\hline 48 - Resolução de problemas & $\mathbf{0 , 7 7 8}$ & 0,234 & 0,235 & 0,001 & 0,116 & 0,140 & 0,749 \\
\hline 56-Restaurante & 0,176 & 0,190 & 0,041 & 0,206 & 0,760 & 0,171 & 0,718 \\
\hline $57-$ Xerox & 0,080 & 0,050 & 0,131 & 0,026 & $\mathbf{0 , 8 8 4}$ & 0,090 & 0,817 \\
\hline 58-Estacionamento & 0,267 & 0,231 & 0,025 & 0,130 & 0,610 & $-0,312$ & 0,612 \\
\hline \% de variância extraída & 35,93 & 11,17 & 8,84 & 7,47 & 6,85 & 3,94 & \\
\hline Autovalor & 7,18 & 2,23 & 1,76 & 1,49 & 1,37 & 0,79 & \\
\hline
\end{tabular}

Fonte: Dados da pesquisa (2013).

A variância total extraída da amostra de validação foi de 71,30\%. A Tabela 2 apresenta a estrutura fatorial, as comunalidades e a variância das dimensões da amostra de validação.

A extração foi realizada através dos autovalores (eigenvalue) superiores a 1, obtendo as seis dimensões propostas na etapa de teste, compostas pelos mesmos indicadores. De acordo com o informado na Tabela 2, na amostra de validação, a comunalidade mínima obtida foi de 0,579 (adequação do currículo à prática) e a máxima de 0,825 (conforto dos ambientes). As cargas fatoriais nas dimensões estiveram entre 0,623 (adequação do currículo à prática) e 0,887 (pesquisa e extensão), não apresentando cargas cruzadas superiores a 0,406 (professores - preparados para aula). 
DOI: http://dx.doi.org/10.5007/1983-4535.2015v8n2p216

Tabela 2 Estrutura Fatorial da Amostra de Validação

\begin{tabular}{|c|c|c|c|c|c|c|c|}
\hline \multirow{2}{*}{ Indicadores } & \multicolumn{6}{|c|}{ Dimensões } & \multirow{2}{*}{ Comunalidades } \\
\hline & 1 & 2 & 3 & 4 & 5 & 6 & \\
\hline 1 - Oferta de aulas práticas & 0,076 & 0,215 & 0,075 & 0,782 & $-0,136$ & 0,201 & 0,728 \\
\hline 2 - Conteúdos $\mathrm{x}$ aulas práticas & 0,168 & 0,198 & 0,070 & 0,746 & 0,274 & $-0,083$ & 0,711 \\
\hline 3 - Adequação do currículo à prática & 0,087 & 0,030 & 0,378 & 0,623 & 0,184 & 0,077 & 0,579 \\
\hline 4 - Ferramentas $p /$ prática profissional & 0,083 & $-0,030$ & 0,239 & 0,659 & 0,331 & 0,010 & 0,608 \\
\hline 5 - Professores - titulação & 0,047 & 0,019 & 0,218 & 0,171 & $\mathbf{0 , 8 0 6}$ & $-0,038$ & 0,730 \\
\hline 6 - Professores - atualizados & 0,127 & 0,218 & 0,060 & 0,144 & $\mathbf{0 , 8 0 8}$ & $-0,091$ & 0,750 \\
\hline 7 - Professores - preparados $\mathrm{p} /$ aula & 0,209 & 0,406 & 0,086 & 0,111 & 0,647 & 0,006 & 0,647 \\
\hline 8 - Serviços à comunidade & $-0,014$ & 0,103 & $\mathbf{0 , 7 3 1}$ & 0,259 & $-0,053$ & 0,094 & 0,624 \\
\hline 9 - Grupos de estudos & 0,054 & 0,092 & $\mathbf{0 , 7 8 8}$ & 0,165 & 0,318 & 0,025 & 0,762 \\
\hline 10 - Projetos de pesquisa ou extensão & 0,090 & 0,070 & $\mathbf{0 , 8 8 7}$ & 0,075 & 0,128 & 0,040 & 0,824 \\
\hline 11 - Salas de aula & 0,194 & 0,815 & 0,040 & 0,125 & 0,126 & 0,032 & 0,737 \\
\hline 12 - Limpeza dos ambientes & 0,286 & $\mathbf{0 , 7 3 9}$ & 0,131 & 0,072 & 0,125 & $-0,004$ & 0,666 \\
\hline 13 - Conforto dos ambientes & 0,183 & $\mathbf{0 , 8 4 9}$ & 0,096 & 0,137 & 0,174 & 0,108 & 0,825 \\
\hline 14 - Eficiência do atendimento & $\mathbf{0 , 7 7 7}$ & 0,198 & 0,147 & 0,034 & 0,161 & $-0,039$ & 0,694 \\
\hline 15 - Comunicação alunos/funcionários & $\mathbf{0 , 8 3 3}$ & 0,254 & 0,010 & 0,083 & 0,009 & 0,143 & 0,787 \\
\hline 16 - Funcionários - educação & $\mathbf{0 , 8 0 8}$ & 0,120 & $-0,021$ & 0,152 & 0,021 & 0,114 & 0,704 \\
\hline 17 - Resolução de problemas & 0,752 & 0,160 & 0,034 & 0,096 & 0,200 & 0,250 & 0,705 \\
\hline 18 - Restaurante & 0,240 & 0,044 & 0,025 & 0,027 & 0,049 & 0,848 & 0,782 \\
\hline $19-$ Xerox & 0,191 & 0,020 & 0,075 & $-0,035$ & $-0,008$ & 0,856 & 0,777 \\
\hline 20 - Estacionamento & $-0,049$ & 0,049 & 0,054 & 0,158 & $-0,147$ & 0,754 & 0,623 \\
\hline \% de variância extraída & 29,25 & 13,14 & 10,78 & 6,37 & 6,11 & 5,62 & \\
\hline Autovalor & 5,85 & 2,62 & 2,15 & 1,27 & 1,22 & 1,12 & \\
\hline
\end{tabular}

Fonte: Dados da pesquisa (2013).

Conforme apresentado, a variância extraída nos fatores apresentou o maior valor na dimensão 1 (29,25\%), seguido por 13,14\%, na dimensão $2,10,78 \%$ na dimensão $3,6,37 \%$ na dimensão 4, 6,11\% na dimensão 5 e 5,62\% na dimensão 6 , compondo os $71,30 \%$ da variação total explicada por esta solução fatorial.

\subsection{ANÁLISE FATORIAL CONFIRMATÓRIA}

Para a análise fatorial confirmatória foi utilizado o software SmartPLS 2.0M3, que utiliza o método de estimação partial least squares e não exige que suposições sobre a distribuição das variáveis sejam atendidas. Além disso, os resultados são robustos quando medidas formativas são utilizadas (RINGLE et al., 2009). Devido à base teórica, este construto é considerado do tipo reflexivo-formativo de segunda ordem (tipo II) (BECKER et al., 2012). O modelo foi rodado com o procedimento factor weighting scheme, apropriado para modelos de mensuração (HENSELER et al., 2009), sendo utilizada a abordagem de indicadores repetidos. Nesta abordagem, a variável latente de segunda ordem é especificada utilizando todas as variáveis manifestas que refletem as variáveis latentes de primeira ordem, ou seja, as variáveis manifestas são utilizadas duas vezes (BECKER et al., 2012). Becker et 
al. (2012) recomendam que essa abordagem seja utilizada com este tipo de construto de segunda ordem.

Primeiramente, foram avaliados os critérios de qualidade para os construtos reflexivos de primeira ordem: confiabilidade, validade discriminante e convergente. As variáveis latentes apresentaram boa confiabilidade. A confiabilidade composta apresentou índices acima de 0,8, e o alpha de Cronbach apresentou índices acima de 0,7, com exceção de corpo docente, que apresentou um índice 0,625, conforme Tabela 3. De acordo com Hair et al. (2009), se todos os outros índices se mostram adequados, é aceitável um alpha de Cronbach de 0,6 .

Tabela 3 Confiabilidade e Validade dos Construtos de Primeira Ordem

\begin{tabular}{l|c|c|c|c|c|c}
\hline & Média & $\begin{array}{c}\text { Desvio- } \\
\text { padrão }\end{array}$ & $\begin{array}{c}\text { Erro- } \\
\text { padrão }\end{array}$ & AVE & $\begin{array}{c}\text { Confiabilidade } \\
\text { composta }\end{array}$ & $\begin{array}{c}\text { Alpha de } \\
\text { Cronbach }\end{array}$ \\
\hline Corpo docente & 0,217 & 0,023 & 0,023 & 0,579 & 0,801 & 0,625 \\
\hline Infraestrutura & 0,292 & 0,021 & 0,021 & 0,754 & 0,902 & 0,837 \\
\hline Org. administrativa & 0,354 & 0,024 & 0,024 & 0,683 & 0,896 & 0,845 \\
\hline Org. curricular & 0,268 & 0,024 & 0,024 & 0,534 & 0,820 & 0,709 \\
\hline Pesquisa e extensão & 0,232 & 0,025 & 0,025 & 0,717 & 0,883 & 0,801 \\
\hline Serviços agregados & 0,140 & 0,033 & 0,033 & 0,707 & 0,877 & 0,792 \\
\hline
\end{tabular}

Fonte: Dados da pesquisa (2013).

A validade convergente das variáveis latentes foi confirmada. Todas as cargas fatoriais ficaram acima de 0,596 e foram significantes. Além disso, todas as variâncias extraídas médias (AVE) ficaram acima de 0,5, conforme Tabela 4. A validade discriminante das variáveis latentes também foi confirmada. Conforme os critérios de Fornell e Larcker (1981), a correlação ao quadrado das variáveis latentes não ultrapassou a AVE, conforme Tabela 4. Além disso, as variáveis manifestas apresentaram cargas fatoriais mais altas em seus respectivos construtos.

Tabela 4 Validade Discriminante dos Construtos de Primeira Ordem

\begin{tabular}{l|c|c|c|c|c|c}
\hline & $\begin{array}{c}\text { Corpo } \\
\text { Docente }\end{array}$ & $\begin{array}{c}\text { Infra- } \\
\text { estrutura }\end{array}$ & $\begin{array}{c}\text { Org. admi- } \\
\text { nistrativa }\end{array}$ & $\begin{array}{c}\text { Org. curri- } \\
\text { cular }\end{array}$ & $\begin{array}{c}\text { Pesquisa e } \\
\text { extensão }\end{array}$ & $\begin{array}{c}\text { Serviços } \\
\text { agregados }\end{array}$ \\
\hline Corpo docente & $0,579^{*}$ & & & & & \\
\hline Infraestrutura & 0,179 & 0,754 & & & & \\
\hline Org. administrativa & 0,135 & 0,236 & 0,683 & & & \\
\hline Org. curricular & 0,160 & 0,139 & 0,096 & 0,534 & & \\
\hline Pesquisa e extensão & 0,144 & 0,048 & 0,044 & 0,188 & 0,717 & \\
\hline Serviços agregados & 0,000 & 0,020 & 0,127 & 0,015 & 0,025 & 0,706 \\
\hline
\end{tabular}

*Os elementos na diagonal representam as AVE das variáveis latentes.

Fonte: Dados da pesquisa (2013). 
Após verificar a confiabilidade e validade das variáveis latentes de primeira ordem, critérios de qualidade para a variável latente de segunda ordem foram averiguados. Por se tratar de um construto formativo, os índices avaliados são os pesos dos indicadores, significância desses pesos e multicolineariedade dos indicadores (BECKER et al., 2012; HAIR et al., 2011). Critérios como confiabilidade e validades convergente e discriminante são inapropriados ou não podem ser avaliados empiricamente (DIAMANTOPOULOS; WINKLHOLFER, 2001; HAIR et al., 2011).

Hair et al. (2011), entretanto, sugerem avaliar a validade convergente com base na análise de redundância. Nesta análise, e acordo com os autores, deve-se correlacionar o construto formativo com uma medida global. Valores acima de 0,800 indicam boa validade convergente. $\mathrm{O}$ item global foi feito com a média do primeiro indicador de cada dimensão. Ao rodar o modelo, este apresentou um índice de 0,892, indicando validade convergente do construto formativo. Quanto aos indicadores, estes apresentaram índices de 0,140 a 0,354. Conforme a Tabela 5, todos os indicadores são significantes. A multicolinearidade foi verificada por meio do fator de inflação da variância (VIF). O índice mais alto de VIF foi de 1,568, indicando baixa multicolinearidade.

Por fim, as validades nomológica e preditiva foram averiguadas utilizando uma abordagem de duas fases, em que os scores das variáveis latentes de primeira ordem são utilizadas em um segundo estágio para o modelo estrutural (BECKER et al., 2012). Os resultados indicam que a qualidade percebida tem uma relação com a intenção de compra ( $\left.b=0,613, t=15,803, p<0,001, R^{2}=0,38\right)$ e com a satisfação do consumidor $(b=0,486, t=9,052$, $p<0,001, R^{2}=0,24$ ), conforme já evidenciado por alguns autores (p. ex., SACHDEV; VERMAN, 2004).

Tabela 5 Critérios de Qualidade para Construto Formativo de Segunda Ordem

\begin{tabular}{l|c|c|c|c}
\hline & Indicador & $\mathbf{T}$ & $\boldsymbol{p}$ & VIF \\
\hline Corpo docente & 0,217 & 9,391 & $<0,001$ & 1,489 \\
\hline Infraestrutura & 0,292 & 14,162 & $<0,001$ & 1,494 \\
\hline Org. administrativa & 0,354 & 14,566 & $<0,001$ & 1,568 \\
\hline Org. curricular & 0,268 & 11,102 & $<0,001$ & 1,430 \\
\hline Pesquisa e extensão & 0,232 & 9,137 & $<0,001$ & 1,337 \\
\hline Serviços agregados & 0,140 & 4,223 & $<0,001$ & 1,193 \\
\hline
\end{tabular}

Fonte: Dados da pesquisa (2013). 


\subsection{ANÁLISE DOS RESULTADOS}

A análise dos dados obtidos através do desenvolvimento desta pesquisa demonstrou que a escala proposta é adequada para a finalidade da mensuração da qualidade percebida em serviços educacionais por alunos de graduação, sendo composta por seis dimensões e 20 indicadores:

1)Organização curricular: Oferta de aulas práticas; Relação entre os conteúdos ministrados e a prática profissional; Adequação do currículo do curso às demandas da prática profissional; Possibilidade de conhecer ferramentas adequadas e atuais para a prática profissional.

2)Corpo docente: Titulação dos professores; Professores atualizados com os conhecimentos práticos e ferramentas da profissão; Professores preparados para as aulas.

3)Pesquisa e extensão: Possibilidade de participar de atividades relacionadas com a prestação de serviço à comunidade; Possibilidade de participar de grupos de estudos; Possibilidade de participar de projetos de pesquisa ou extensão.

4)Organização administrativa: Eficiência no atendimento; Comunicação entre alunos e funcionários; Educação dos funcionários; Resolução de problemas.

5)Infraestrutura: Salas de aula; Limpeza dos ambientes; Conforto dos ambientes.

6)Serviços agregados: Restaurante; Xerox; Estacionamento.

\section{CONSIDERAÇÕES FINAIS}

Os resultados desta pesquisa apontam a confiabilidade e validade da escala proposta, demonstrando sua adequação à finalidade desta pesquisa. A literatura analisada, em sua maioria, aponta os indicadores e características da qualidade percebida em serviços de forma genérica, sem atentar para as especificidades do contexto em que o serviço está inserido (PARASURAMAN et al., 1985; ZEITHAML, 1988; CRONIN; TAYLOR, 1992). Diante disso, cabe destacar o caráter inovador da escala proposta nesta pesquisa, uma vez que foi desenvolvida com a finalidade de compreender as particularidades do fenômeno qualidade percebida, no contexto específico do ensino superior.

Além das contribuições acadêmicas este trabalho apresenta relevantes implicações relacionadas às práticas gerenciais, uma vez que oferece uma alternativa em relação à forma como a mensuração da qualidade percebida é realizada atualmente no ensino superior. 
Através da aplicação da escala de forma regular, será possível definir índices de qualidade percebida nas IES, permitindo comparar os resultados obtidos entre as instituições, identificando seu desempenho em relação a concorrentes além de acompanhar a evolução e percepção por parte dos alunos em relação às melhorias desenvolvidas pelas instituições. Também será possível identificar a qualidade percebida por alunos de diferentes níveis e cursos, permitindo a tomada de decisão focada no perfil e necessidades específicas dos mais variados perfis.

Pode-se identificar limitações para este estudo, sem que haja comprometimento das implicações acadêmicas e gerenciais apresentadas. Uma primeira limitação refere-se ao fato da pesquisa ter sido aplicada somente em duas IES, sem que haja a ampliação para outros grupos e cidades. Além disso, a opção pela técnica de amostragem não-probabilística, por conveniência, limita a generalização dos resultados para toda a população. Diante das limitações apresentadas, sugere-se que estudos posteriores realizem a aplicação desta escala em diferentes amostras, buscando garantir uma maior variação no perfil dos respondentes.

Ainda, pode-se identificar a necessidade de incluir os demais stakeholders envolvidos com a prestação do serviço educacional no modelo proposto. Srikanthan e Dalrymple (2003) apontam que os modelos de qualidade adotados, em relação aos serviços educacionais, até o momento, falham em não envolver os principais grupos de relacionados com o ensino superior. O ideal é um modelo que seja sensível às expectativas dos diferentes grupos de pessoas envolvidas, sugerindo os fornecedores, alunos, ex-alunos e funcionários. Desta forma, sugere-se ampliar, em estudos futuros, o estudo envolvendo as partes envolvidas.

Em relação à escala, sugere-se sua efetiva aplicação nas IES, possibilitando a qualificação da gestão através da identificação da percepção da qualidade por parte de seus alunos, permitindo melhorias necessárias aos serviços entregues pelas IES, garantindo aumento de sua competitividade.

\section{REFERÊNCIAS}

BANDEIRA, M. L.; GONÇALVES, C. A.; VEIGA, R. T.; HUERTAS, M. K. Z. Avaliação da qualidade do ensino de pós-graduação: elementos para a construção e validação de um instrumento de pesquisa. In: Encontro Nacional da Associação Nacional dos Programas de Pós-Graduação em Administração (ENANPAD), 22., 1998, Foz do Iguaçu. Anais... Rio de Janeiro: ANPAD, 1998.

BASSO, K.; SCHWAB, E. A.; PÓLVORA, R. P.; MARQUES, L. T.; PEREIRA, M. C.; SLONGO, L. A. Relações de interdependência entre confiança, comprometimento, qualidade 
percebida e lealdade em estudantes do ensino superior. In: Encontro de Marketing (EMA), 3., 2008, Curitiba. Anais... Rio de Janeiro: ANPAD, 2008.

BECKER, J. M.; KLEIN, K.; WETZELS, M. Hierarchical latent variable models in PLSSEM: Guidelines for using reflective-formative type models. Long Range Planning, v. 45, p. 359-394, 2012.

BORGES JR, A. A.; FONSECA, M. J. O uso da pesquisa de satisfação do consumidor como instrumento de política pública: o potencial de uso no caso do transporte coletivo de Porto Alegre. RIMAR - Revista Interdisciplinar de Marketing, v. 1, n. 3, p. 38-50, 2002.

BRASIL. Constituição (1988). Constituição da República Federativa do Brasil. Brasília: Senado, 1988.

CARMAN, J. M. Consumer perceptions of service quality: an assessment of the SERVQUAL dimensions. Journal of Marketing, v. 66, n. 1, p. 33-35, 1990.

CASAGRANDE, R. A avaliação institucional voltada às perspectivas estratégicas da organização. In: COLOMBO, S. S.; RODRIGUES, G. M. (org.). Desafios da Gestão Universitária Contemporânea. Porto Alegre: Artmed, p. 219-243, 2011.

CASTANHEIRA, A. M. P.; COELHO, A. R.; GAGLIARDI, M. V. Avaliação Institucional: ações consolidadas na graduação. São Paulo: Editora Mackenzie, 2008.

CERCHIARO, I. B.; MOTA, M. C. Avaliação da qualidade do serviço educacional numa IES particular: a visão do aluno de graduação sobre a qualidade percebida. In: Encontro de Marketing (EMA), 4., 2010, Florianópolis. Anais... Florianópolis: ANPAD, 2010.

CHURCHILL, G. A. J. A paradigm for developing better measures of marketing constructs. Journal of Marketing Research, v. 16, p. 64-73, 1979.

CRONIN, J.; TAYLOR, S. Measuring service quality: a reexamination and extension. Journal of Marketing, v. 56, n. 3, p. 55-68, 1992.

CRONIN, J.; TAYLOR, S. SERVPERF versus SERVQUAL: reconciling performance-based and perceptions-minus-expectations measurement of service quality. Journal of Marketing, v. 58, p. 125-131, 1994.

DIAMANTOPOULOS, A.; WINKLHOLFER, H. M. Index construction with formative indicators: an alternative to scale development. Journal of Marketing Research, v. 38, n. 2, p. 269-277, 2001.

FORNELL, C.; LARCKER, D. F. Evaluating structural equation models with unobservable variables and measurement error. Journal of Marketing Research, v. 18, n. 1, p. 39-50, 1981.

FREITAS, A. L. P.; RODRIGUES, S. G. A estruturação do processo de auto-avaliação de IES: uma contribuição para gestão educacional. In: Encontro Nacional de Engenharia de Produção (ENEGEP), 23., 2003, Ouro Preto. Anais... Ouro Preto: ENEGEP, 2003. p. 1-8. 
GARCIA, M. As mudanças no cenário do ensino superior privado. In: GARCIA, M. (org.). Gestão profissional em instituições de ensino superior: um guia de sobrevivência para mantenedores, acionistas, reitores, pró-reitores, diretores, coordenadores, gerentes e outros gestores institucionais. Vila Velha: Editora Hoper, p. 15-19, 2006.

HAIR, J. F.; RINGLE, C. M.; SARSTEDT, M. PLS-SEM: indeed a silver bullet. Journal of Marketing Theory and Practice, v. 19, n. 2, p. 139-151, 2011.

HAIR, J. F.; TATHAM. R. L.; ANDERSON, R. E.; BLACK, W. Análise multivariada de dados. 6. ed. Porto Alegre: Bookman, 2009.

HENNIG-THURAU, T.; LANGER, M. F.; HANSEN, U. Modeling and managing student loyalty: an approach based on the concept of relationship quality. Journal of Service Research, v. 3, n. 4, p.331-344, 2001.

HENSELER, J.; RINGLE, C. M.; SINKOVICS, R. R. The use of partial least squares path modeling in international marketing. Advances in International Marketing, v. 20, p. 277-319, 2009.

INEP - INSTITUTO NACIONAL DE ESTUDOS E PESQUISAS EDUCACIONAIS. Evolução da Educação Superior - Graduação. Instituto Nacional de Estudos e Pesquisas Educacionais. Disponível em $<\underline{\text { http://portal.inep.gov.br/web/censo-da-educacao- }}$ superior/evolucao-1980-a-2007>. Acesso em: 23 abr. 2013.

KOTLER, P.; FOX, K. F. A. Marketing estratégico para instituições educacionais. São Paulo: Atlas, 1994.

LAZZARI, F.; MILAN, G. S.; EBERLE, L. A identificação das dimensões da qualidade dos serviços prestados por laboratórios universitários e a satisfação de clientes. In: Seminários de Administração (SEMEAD), 12., 2009, São Paulo. Anais... São Paulo: FEA/USP, 2009.

LOPES, E. L.; HERNANDES, J. M. C.; NOHARA, J. J. Escalas concorrentes para a mensuração da qualidade percebida: uma comparação entre a SERVQUAL e a RSQ. Revista de Administração de Empresas - RAE, v. 49, n. 4, p. 401-416, 2009.

LOURENÇO, C. D. S.; KNOP, M. F. T.; OLIVEIRA, V. C. S.; SILVA, M. R. J. D. Ensino superior em administração e percepção da qualidade de serviços: uma aplicação da escala SERVQUAL. In: Encontro Científico de Administração, 30., 2006, Salvador. Anais...

Salvador: ANPAD, 2006.

MALHOTRA, N. Pesquisa de marketing: uma orientação aplicada. 6. ed. Porto Alegre: Bookman, 2012.

MEYER, V.; SERMANN, L. I. C.; MANGOLIM, L. Planejamento e gestão estratégica: viabilidade na IES. In: Congresso Nacional da Área da Educação (EDUCERE), 4., 2004, Curitiba. Anais... Curitiba: PUC/PR, 2004. 
MIGUEL, P. A. C.; SALOMI, G. E. Uma revisão dos modelos para medição da qualidade em serviços. Revista Produção, v. 14, n. 1, p. 12-30, 2004.

MOURA, L. R. C.; SALDANHA, L. C. L.; VEIGA, R. T.; MOURA, L. E. L.; CUNHA, N. R. S. Avaliação da qualidade de serviços utilizando a escala SERVQUAL: o estudo de caso Matermed. Reuna: Belo Horizonte, v. 12, n. 3, p. 21-36, 2007.

PARASURAMAN, A.; ZEITHAML, V. A.; BERRY, L. A conceptual model of service quality and its implications for future research. Journal of Marketing, v. 49, p. 41-50, 1985.

PARASURAMAN, A.; ZEITHAML, V. A.; BERRY, L. A multiplem-item scale for measuring consumer perceptions of service quality. Journal of Retailing, v. 64, n. 1, p. 12-40, 1988.

PIVETTA, H. M. F.; BACKES, D. S.; CARPES, A.; BATTISTEL, A. L. H. T.; MARCHIORI, M. Ensino, pesquisa e extensão universitária: em busca de uma integração efetiva. Linhas Críticas, v. 16, n. 31, p. 333-390, 2010.

RINGLE, C. M.; GÖTZ, O.; WETZELS, M.; WILSON, B. On the use of formative measurement specifications in structural equation modeling: A Monte Carlo simulation study to compare covariance-based and partial least squares model estimation methodologies. In: METEOR Research Memoranda (RM/09/014): Maastricht University, 2009.

ROSSITER, J. R. The C-OAR-SE procedure for scale development in marketing. International Journal of Research in Marketing, v. 19, p. 305-335, 2002.

SACHDEV, S. B.; VERMA, H. V. Relative importance of service quality dimensions: a multisectoral study. Journal of Services Research, v. 4, n. 1, p. 93-116, 2004.

SIQUEIRA, R.; CARVALHO, J. Qualidade do serviço educacional prestado por escolas de administração: confronto entre uma universidade pública e uma faculdade privada. In: Encontro da Associação Nacional de Pós-Graduação e Pesquisa em Administração (ENANPAD), 3., 2006, Salvador. Anais... Salvador: ANPAD, 2006.

SRIKANTHAN, G.; DALRYMPLE J. Developing alternative perspectives for quality in higher education. The International Journal of Education Management, v. 17, n. 3, p. 126136, 2003.

YUE, G. K.; MAÑAS, A. V. Prestação de serviços: um estudo sobre a percepção da qualidade do usuário em telefonia fixa na cidade de São Paulo. Integração, v. 11, n. 42, p. 205-217, 2005.

ZEITHAML, V. A. Consumers perception of price, quality, and value: a means-end model and syntesis of evidence. Journal of Marketing, v. 52, p. 2-22, 1988.

ZEITHAML, V. A. Service quality, profitability, and the economic worth of costumers: what we know and what we need to learn. Journal of the Academy of Marketing Science, v. 28, n. 1, p. $67-85,2000$. 